\title{
An artificial neural network-based diagnostic methodology for gas turbine path analysis-part I: introduction
}

\author{
Roberto Capata ${ }^{1}$ \\ ${ }^{1}$ Department of Mechanical and Aerospace Engineering, University of Roma "Sapienza”, Rome, Italy
}

Received: 21 September 2015/Revised: 9 September 2016/Accepted: 10 September 2016/Published online: 28 September 2016 (c) Joint Center on Global Change and Earth System Science of the University of Maryland and Beijing Normal University and Springer-Verlag Berlin Heidelberg 2016
Abstract The reliability of gas path components (com-
In the case of components failures, the system provides pressor, burners and turbines) of a gas turbine is generally high, when compared with those of other systems. How- ever, in case of forced stops, downtime is usually high, with a relatively low availability. The purpose of condition monitoring and fault diagnostics is to detect, isolate and evaluate (i.e., to estimate quantitatively the extent) defects within a system. One effective technique could provide a significant improvement of economic performance, reduce operating costs and maintenance, increase the availability and improve the level of safety achieved. However, con- ventional analytical techniques such as gas path analysis and its variants are limited in their engine diagnostic, due to several reasons, including their inability to effectively operate in the presence of noise measures, to distinguish anomalies of component from a failure sensor, to preserve the linearity in the relations between parameters of gas turbines and to manage the sensors range to achieve accurate diagnosis. In this paper, the approach of a diag- nostic scenario to detect faults in the gas path of a gas turbine has been presented. The model provides a large- scale integration of artificial neural networks designed to detect, isolate and evaluate failures during the operating conditions. The engine measurements are considered as input for the model, such as the speed, pressure, tempera- ture and fuel flow rate. The output supplies any changes in the sensor or in the efficiency levels and flow rate, in the event of fault components. The diagnostic method has the ability to evaluate anomalies of both multiple components and multiple sensors, within the range of operating points.
Roberto Capata
roberto.capata@uniroma1.it diagnostic changes in efficiency and flow rate that can be interpreted to determine the nature of the physical problem. The technique has been applied in different operating conditions by comparing the results obtained with the solutions provided by linear and nonlinear analysis.
Keywords Gas turbine diagnostic - Gas path analysis . Neural network

\section{List of symbols}
$a \quad$ Correction exponent for $\delta$
$a \quad$ Prediction of a network performance parameter
ADT Advanced diagnostic techniques
ANN Artificial neural network
$b \quad$ Correction exponent for $\theta$
$c \quad$ Correction exponent for $\gamma$
CoC Coefficient of correlation
DOD Direct object damage
DSA Double-step analysis
FL Fuzzy logic
FMF Fuel mass flow rate
FOD Foreign object damage
GG Gas generator
GPA Gas path analysis
GPF Gas path failure
GPFD Gas path fault diagnosis
GT Gas turbine
HCF High-cycle fatigue
KF Kalman filter
LCF Low-cycle fatigue
MBD Model-based diagnostic
MMKF Multimodeling Kalman filter 
$n \quad$ Number of engine measurements

$N \quad$ Relative velocity, number of tests

p Pressure

$\mathrm{P} \quad$ Power turbine

$r \quad$ Number of measurements that can be considered as disturbed measurements

RNA Ribonucleic acid

SF Sensor fault

SL Sea level

SP Shaft power

$T \quad$ Temperature

TBO Time between overhaul

TSA Triple step analysis

TIT Turbine inlet temperature

$X, Z, z \quad$ Input parametrs

\section{Greek symbol}

\section{$\eta \quad$ Efficiency}

$\Gamma \quad$ Flux capacity of the function/component

$\sigma \quad$ Standard deviation

$\gamma \quad$ Power ratio between shaft power and reference/ design power

$\delta \quad$ Pressure ratio at suction side

$\rho_{\mathrm{m}} \quad$ Measured parameter value at environmental pressure, temperature and rated power

$\theta \quad$ Temperature ratio between the environmental temperature and reference temperature

$\Delta \quad$ Difference

\section{Subscripts}

a Ambient

bki Corrected reference for sensor " $i$ " on " $k$ " model

C Compressor

cki Network prevision for sensor " $i$ " on " $k$ " model

CT Compressor driven by turbine

i Sensor

ki K model sensor

PT Power turbine

$\mathrm{T}$ Turbine

\section{Introduction}

The turbine plays an increasingly important role in the energy generation and distribution. This aspect leads to improve economic performance, by improving the efficiency of components, extending the periods between maintenance (TBOS) and increasing the overall performance. The cost per kilowatt value produced by a GT (€/ $\mathrm{kW}$ ) is one of the most aspect in the plant management and includes all necessary costs to maintain the turbine operational. The efficiency variation should be considered in the calculation of the plant life cycle. The availability of gas path components might be relatively low because high downtime is normally associated with forced stops (Agrawal et al. 1978). A possible solution to increase the availability is to improve the maintenance operations, based on monitoring conditions (CBM). Before the advent of the GPA (gas path analysis) tools, the gas path failures have been diagnosed mainly by means of quality tools, by matching symptoms to pre-established patterns (to identify the tendency to the variation in the engine measurements and then isolate a failing module or component). This approach does not consider that the apparent fault could be due to measurement noise. The solutions cannot be provided by qualitative approaches, both conventional and evolutionary. GPA and its variants, as well as some artificial intelligence techniques, are able to provide quantitative results for the breakdowns in the gas path.

\section{Methodology briefings}

\subsection{Gas path fault}

A GT plant is exposed to several failures as reported in Table 1 (Agrawal et al. 1978; Aker and Saravanamuttoo 1989; Anderson and McNeill 1992). A failure in the gas path (GPF) can be described by directional changes in the size or can be diagnosed by their effect on performance independent variables. It is not possible to describe all possible defects that affect the GT gas path. Table 1 reports the most common failures that can produce a significant efficiency loss or dangerous stops, during the operational duty.

\subsection{Conventional approaches}

The classification of conventional approaches includes all diagnostic techniques that compare the symptoms with preestablished models (Agrawal et al. 1978; Aker and Saravanamuttoo 1989; Ali and Gupta 1990; Allwood et al. 1996; Anderson and McNeill 1992), to identify the tendency to the variation in the measurements of the engine and isolate a faulty module or component.

\subsection{Analytical approaches}

This method uses gas path engine detectable data (dependent variables) to determine the variations of component characteristics and the flow rate (independent variable/ primary performance). This change in operating characteristics can be caused by a failure of a component or a module. Consequently, a potential source of mismatch is the "not repeatability of the measure," due to the operating environment noise. The primary use of dependent 
parameters, for isolating GPF, is based on the fact that the primary performance parameters or independent variables are not directly measurable. They are related to the thermodynamically dependent variables, so that changes in the latter ones are induced by changes in independent variables, with the possibility to identify the failing component. The implementation of the GPA as analytical system has been limited by the component sensors that intrinsically lack of strength. In fact, their positions inside the turbine, often, are not optimal. Their selection is ineffective compared to the failures matrix (Agrawal et al. 1978; Barschdorff 1991). This interconnection matrix is required to identify the various failures (Agrawal et al. 1978). In response to some of these sensor problems, GPA programs, designed to operate with large performance deviations, have been realized.

\subsection{Evolutionary approaches}

To create possible functions with some degrees of freedom, and develop high-level systems, researchers have adopted some tools related to the biological sciences. Artificial intelligence (AI) is divided into several areas such as expert systems, fuzzy logic, genetic algorithm and artificial neural networks (Ali and Gupta 1990; Angelakis et al. 2001; Barschdorff 1991). Among these, the artificial neural networks (ANNs) have been shown to respond to the needs by offering the "quasi-optimal" interpretation of symptoms. The possibility of integrating the artificial neural networks (ANNs) in plant monitoring has recently been investigated by several researches (Bakal et al. 1995; Barlett and Uhrig 1992; Shalan et al. 2010; Hajagos and Bérubé 2001; Centeno et al. 2005) It can be remembered that an artificial neural network is described as a schematic representation of a mathematical equation that receives values (inputs) and generates results (output). The nonlinearity in the performance of the GT and limits of GPA analytical techniques-based model makes inevitable the need for interpretation by ANN model. The basic steps needed to obtain a typical supervised feed-forward ANN include:

1. Evaluate the problem to be solved, in an attempt to verify the possibility of discretization.

2. Generation of test data and training.

3. Definition and formation of various network architectures, to obtain the optimum one. The training process disrupts the weights and variations, to generate the collection that optimizes the system performance, with reduced errors and good generalization. The "Weight adjustment" for the case of a counter reaction network, which operates on the gradient decrease techniques, is achieved by:

$$
\Delta w_{i j}=\eta\left(-\frac{\partial E(n)}{\partial w_{i j}}\right)+\alpha \Delta w_{i j}(n-1)
$$

4. Try the ANN with enough data, to determine the generalization ability.

\section{Advanced diagnostic methodology}

\subsection{Advanced diagnostic techniques (ADT) for gas path fault diagnosis (GPFD)}

In this research, an engine model operating in steady-state conditions will be considered. For the GT presented, a nonlinear simulation package to assemble the models is used. The engine decreasing performance is modeled by scale factor adjustments of efficiency and flow rate of the considered component (ith). The aim is to use these templates to generate data for networks training and testing. An effective application of the developed method should avoid the use of engine model, once completed the networks training, because the parameters have been already fixed. In this situation, our

Table 1 Gas turbine fails and failures

\begin{tabular}{lllll}
\hline Compressor & Turbine & Combustor & Filter & Mechanical problems \\
\hline Fouling & Fouling & Corrosion & Fouling & Bearings problems \\
Blade fatigue & Corrosion & Cracking & Clogging & Critical speeds \\
Blade pitting, blade corrosion & Hot corrosion, sulfidation & Nozzle imbalance & Icing & Unbalance \\
surge & FOD/DOD & Fuel quality & Airflow distortion & Looseness and misalignment \\
Corrosion, erosion & Excessive back pressure & Vibration and pulsation & Humidity effects & Foundations \\
LCF/HCF & Creep & Clogging & Loss of airtightness & Rotor bows \\
FOD & Nozzle bowing & Leakages & & \\
& Bearings distress & Fretting corrosion & & \\
& HCF/LCF & & & \\
& Blade coating & & & \\
\hline
\end{tabular}


application of ANN can be seen as based on learning from examples, rather than based on diagnostics (MBD).

\subsection{Sensors}

The system performances are detected by sensors. These are usually smaller than the number of variables to be monitored. As a result, computational level, the system possesses infinite degrees of freedom to obtain the solution. In order to reach a solution "correct and probable," it is important to establish boundary conditions. Also the choice of sensor type and its placement is fundamental in the creation of the array. Therefore, the operating conditions must be "observed" unambiguously. One possible method for choice of sensors and their location may be to simulate a fault and collect data from the instruments. In this way, it is possible to determine the optimal combination, able to measure the dependent variables that describe the phenomenon or failure. In general, this generates a number of possible combinations of measure sets, with each series that can diagnose similar failures in gas path. In Table 2, a list of proposed sensors for a GT system is given. In addition, each sensor and device has its reference line (operation line under varying inlet conditions). The variation of the measured values from this reference line is calculated by Eq. (2). The equation is applicable to both independent and dependent variables.

$\Delta \%=\frac{Z-Z_{\text {estbaseline }}}{Z_{\text {estbaseline }}} \cdot 100$

$Z_{\text {estbaseline }}$ is the basic condition set, and $Z$ is the measured or calculated value.

The non-repeatability of the instrument is excluded, that is applied as white noise with a Gaussian distribution, to generate the engine data in the range of three standard deviations $(3 \sigma)$ of the mean value.

\subsection{Parameters correction}

To evaluate the corrections, several systems have been simulated. The operating point is determined by three parameters, as well as the shaft power $(P)$, the ambient temperature $\left(T_{\mathrm{a}}\right)$ and pressure $\left(p_{\mathrm{a}}\right)$. These parameters can vary during the plant operation. The aim is to obtain reference measurements, to generate the reference line. It can be noticed that a diagnostic program should require that the engine data are generated whenever a fault diagnosis is carried out, or find alternative methods to obtain these reference lines (Bakal et al. 1995; Hajagos and Bérubé 2001; Centeno et al. 2005). In addition, an analytical approach, derived by the TSA procedure (triplestep analysis), has been used. The approach utilizes a database of various operating points (available in literature and by experimental tests (Capata 2015)) on which a three-dimensional interpolation can be realized, to determine the baseline for each engine measures, after setting the current value to the environment conditions and power (Allwood et al. 1996; Bakal et al. 1995; Barschdorff 1991). A correct definition and positioning of the reference line is a step toward obtaining a sufficiently accurate value for the deviations of each measurement (Allwood et al. 1996). The results show that this process is fast and reliable for regions with operational points distributed on the component operative maps. Moreover, a relationship between the engine measurements and the operating point parameters is derived, if any. So, the region where to concentrate the TSA data correction is obtained, and finally, the TSA method for parameters correction is described (Anderson and McNeill 1992) and shown in Fig. 1. In fact, for each obtained engine measure, and for each point on the surface obtained from various combinations of temperature $\left(T_{\mathrm{a}}\right)$ and pressure $\left(P_{\mathrm{a}}\right)$, it can define some relationships, such as to transform that base point to another reference point, arbitrarily and previously defined. By extension, any surface, defined by a different engine conditions, probably caused by the deterioration of a component or system, can be sufficiently described by relations developed to the reference line. Since the surfaces are irregular, it is reasonable to consider the fact that different points on the surface would require several exponents of correction, a high exponent value for a positive slope and a lower value for a negative slope.

\subsection{Relationship between operating point parameters and engine data}

In order to make the corrections, different configurations of GT plant were simulated. Among these, the simulations
Table 2 Sensors, identity and accuracy

\begin{tabular}{llll}
\hline Sensor & Identity & Unit & $\begin{array}{l}\text { (\%) Required accuracy } \\
\text { over span }\end{array}$ \\
\hline$n_{1}$ & Speed & RPM or 1/s & \pm 0.03 \\
$p_{2}$ & Compressor outlet pressure & Atm. & \pm 0.1 \\
$T_{2}$ & Compressor outlet temperature & $\mathrm{K}$ & \pm 0.4 \\
FMF & Fuel flow & $\mathrm{kg} / \mathrm{s}$ & \pm 1.0 \\
$p_{4}$ & Exhaust pressure & Atm. & \pm 0.1 \\
$T_{4}$ & Exhaust temperature & $\mathrm{K}$ & \pm 0.4 \\
\hline
\end{tabular}


Fig. 1 Analytical approach of dual pitch correction for various environmental conditions

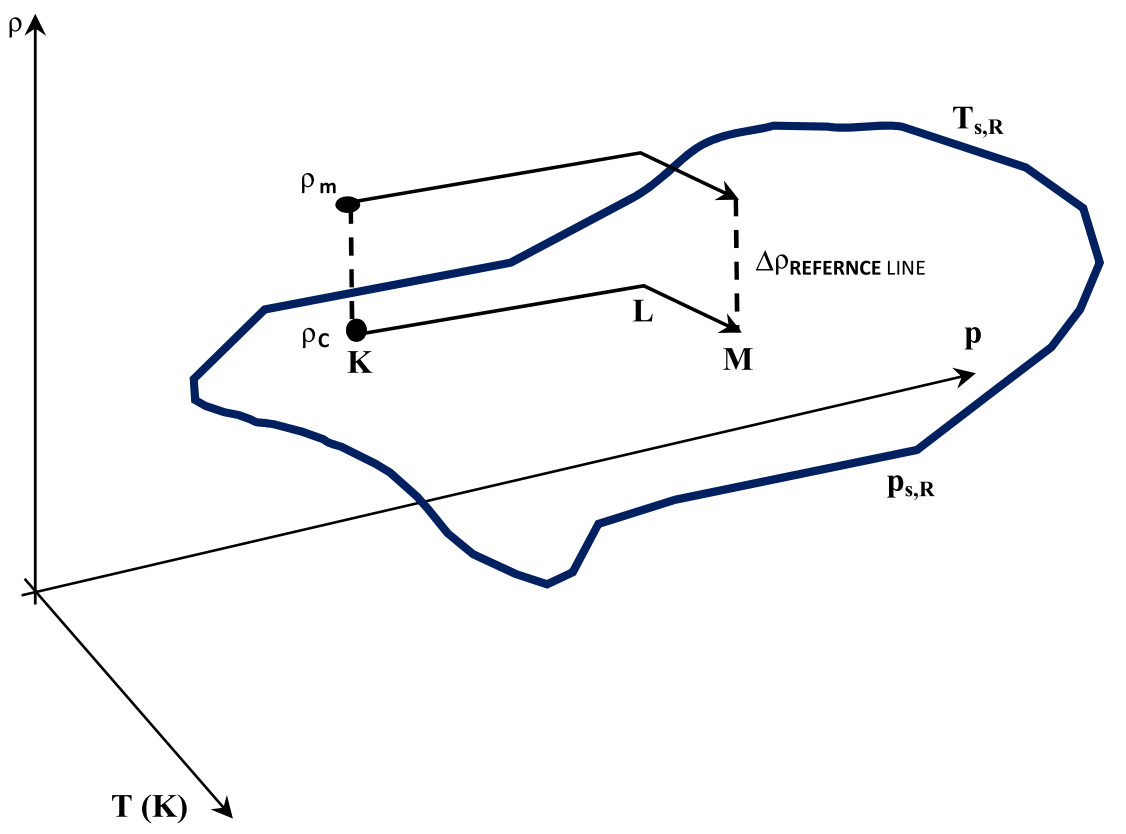

conducted (using various simulation codes as GateCycle, Aspen, MATLAB (Colombo et al. 2003) and a software developed in the Department-Camel) on a gas turbine, with the operating parameters shown in Table 3. This is because the actual operating values were available (stationary, off design and stops). In addition, before delving into the calculation of exponents for the variables that define the operating point $\left(P, p_{\mathrm{a}}\right.$ and $\left.T_{\mathrm{a}}\right)$, it is need to establish if a relationship exists between these variables and the engine data collected (Barlett and Uhrig 1992). All data shown as an increase in the shaft power $(P)$ generate an increase in all measures. In fact, during the nominal operating conditions, a power shaft increase, without any engine failures, should produce an increase in the rotational speed, pressure and temperature. Analogously, maintaining a constant shaft power $(P)$ and $p_{\mathrm{a}}$ and increasing $T$, the engine operates at higher temperature, increases the speed and mass flow and decreases the stage pressure. The fuel flow rate decreases with increasing temperature and decreasing combustor temperature. Finally, increasing $p_{\mathrm{a}}$, stage pressure increases, while decreasing other parameters (Figs. 2, 3).

In Fig. 4 (Ogaji 2003), the variations of the three setup point parameters are shown simultaneously, comparing to the relative speed of the shaft and the low-pressure GT. The figure shows that the speed of the shaft is higher at low pressures and high temperatures while the lowest point is at

Table 3 GT operational parameters

\begin{tabular}{ll}
\hline Mass flow rate $[\mathrm{kg} / \mathrm{s}]$ & 75 \\
Pressure ratio & 8.5 \\
TIT $[\mathrm{K}]$ & 1350 \\
\hline
\end{tabular}

low temperatures and high pressures (Anderson and McNeill 1992; Angelakis et al. 2001). The reason for this is that the compressor work varies with environmental conditions, because it is easier to compress the cold air than hot air. Moreover, in hot conditions, the density varies and the only way to produce the same power level is to increase the mass flow rate through the motor shaft, which rotates faster. Besides, the various surfaces in the figure represent varying levels of power setting.

Considering the relationship between the measurements and the parameters, it is evident that these relationships are not linear. In addition, surface charts in Fig. 3 show that each surface has a form of distortion that makes it not parallel to those nearby, but identical between them.

The regression analysis conducted to map points on these surfaces failed. In fact, it was necessary to apply separate coefficients for each surface. So, the approach with adaptive TSA coefficients was considered and designed. The TSA approach determines, first of all, the exponents for each measurements of the base surface and then applies these exponents to calculate the surface deterioration. Final step is the needed data correction.

\subsection{Basic assumption}

For each assigned engine operational point, generated by variations of the components characteristics, the surface obtained, varying the environmental temperature, pressure and rated power, is considered identical to its base surface (obtained without presence of engine failure). This area is located on a different plane, due to the difference of measurement lines (Fig. 5). In fact, the "degraded 
Fig. 2 Gas path principles (Agrawal et al. 1978)
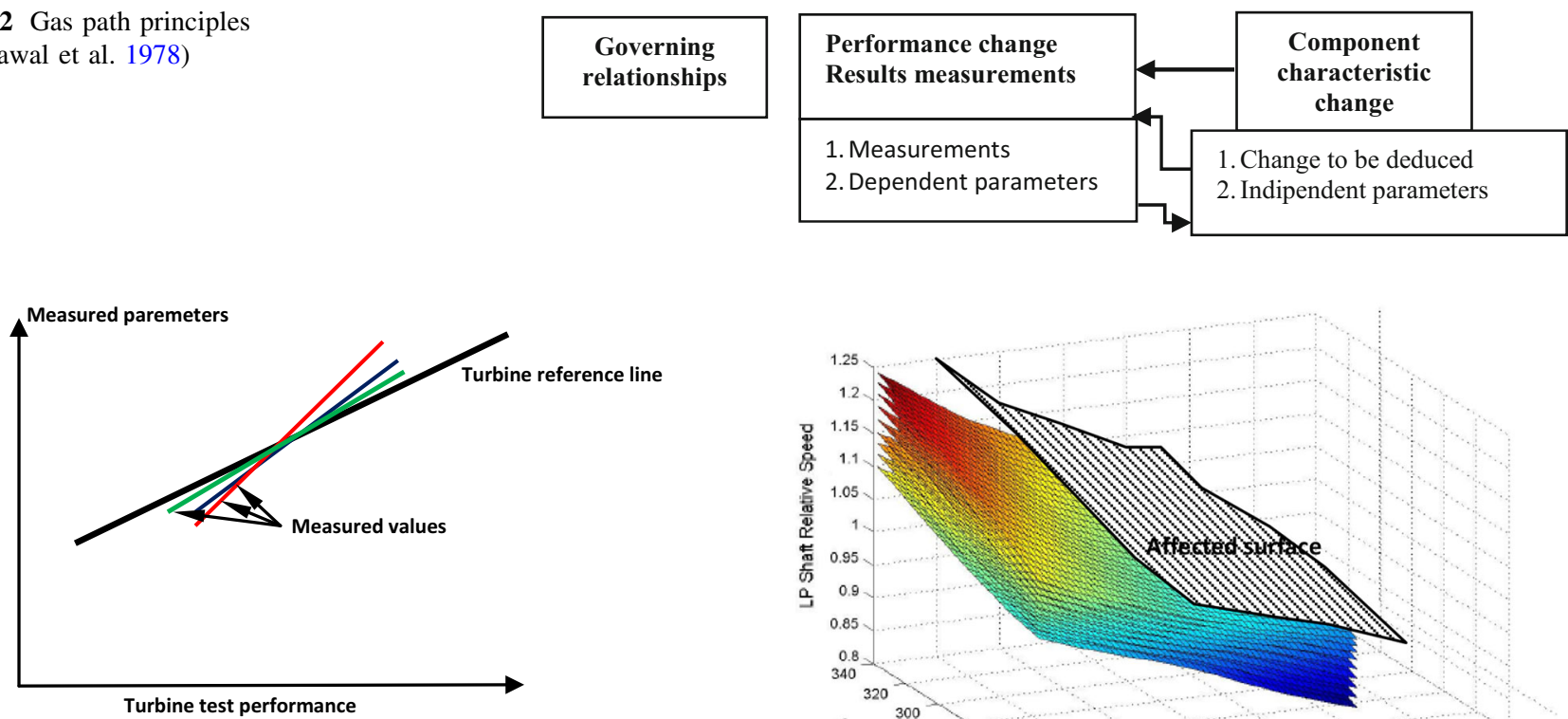

Fig. 3 Comparison between component/engine reference line and measured parameters

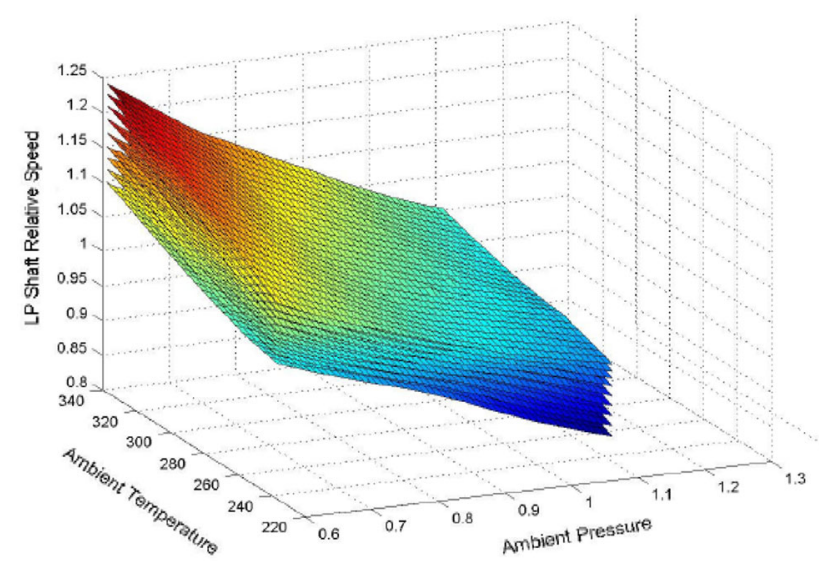

Fig. 4 Effect of $p_{\mathrm{a}}, T_{\mathrm{a}}$ and SP variation on the shaft relative speed

conditions" can be represented by this new surface. Analyzing both figures, it can reasonably consider valid the precondition on the various surfaces similarity, at different failure levels within the operating range. Repeating the construction for all parameters, it can be notice that, once again, the surface curvature similarity is clear and seems to validate our assumptions. A method to validate our assumptions is to determine the percentage deviation between the scenarios, obtained in the presence of an engine fault, with that in the absence of failure, through a given operating range for each parameter $\rho$ (Ogaji 2003).

In this case, Eq. (3) is applied.

$\%$ Deviation $=\frac{\rho_{\text {measured }}-\rho_{\text {baseline }}}{\rho_{\text {baseline }}} \cdot 100$

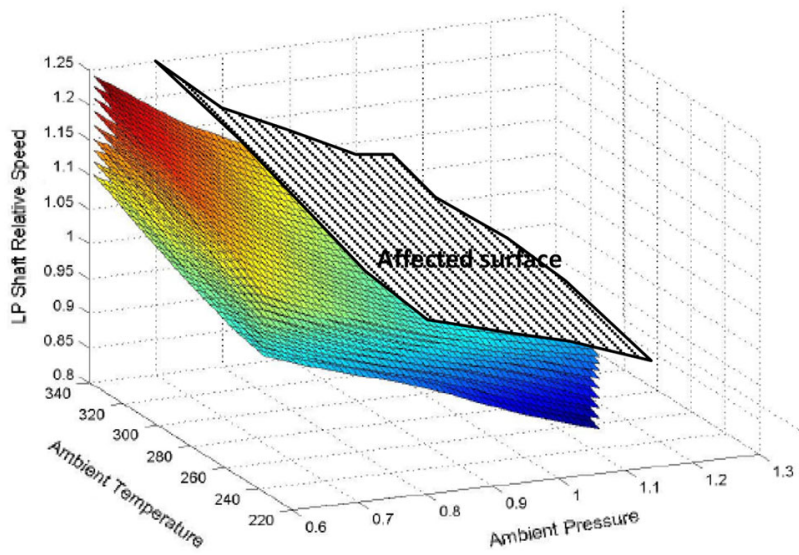

Fig. 5 Operational and affected system surfaces

The equation provides a relative comparison, which allows to verify the consistency changes throughout the range adopted.

\section{The proposed "ANN" methodology}

The processes, used for performing the parameters corrections, can be basically divided into two levels:

Level 1: Determine the correction exponents from the reference surface in ambient conditions and the rated power. This multidimensional problem requires three steps. Level 2: Use the exponents obtained for correct engine measures at sea level-reference point specified.

\subsection{Level 1}

The TSA approach can actually be carried out in any of six possible routes of which we introduce one, i.e., form point $\mathrm{K}$ to $\mathrm{L}$ and then to $\mathrm{M}$, which is a gradual correction of pressure, temperature, and then finally to shaft power (note that the MN is not indicated for simplicity). This is shown in Fig. 5.

Consider the surface in Fig. 3, and report it in Fig. 6. The details are:

1. Given a point $\mathrm{K}$ with environmental pressure and temperature $p_{\mathrm{ak}}$ and $T_{\mathrm{ak}}$, respectively, it generates a calculated value of the parameter considered $\rho_{\mathrm{c}}$, through an interpolation from a database or from an 


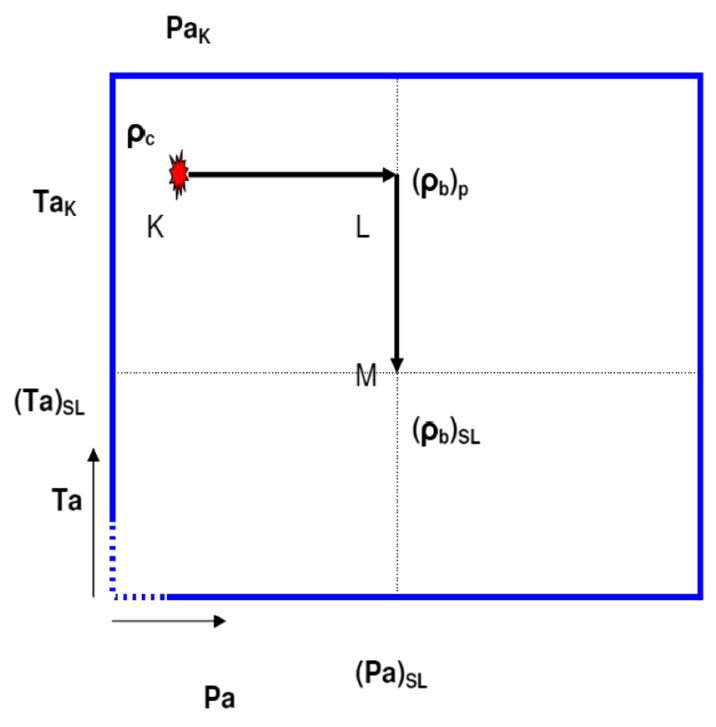

Fig. 6 DSA approach on a reference surface (Ogaji 2003)

accurate thermodynamic engine model (previously mentioned), in relation to environmental temperature and pressure. Both approaches should give roughly the same results because the database was obtained from engine model. However, any difference between the results could be due to errors of approximation.

2. From the surface, using a $2 \mathrm{D}$ interpolation, it obtains a value for the parameter in section $\mathrm{L}\left(\rho_{\mathrm{b}}\right)_{p}$, for example, the pressure value SL but the temperature remains at $T_{\mathrm{ak}}$.

3. Subsequently, with the same technique as described above, a reference value for $\mathbf{M}\left(\rho_{\mathrm{b}}\right)$ is obtained. It is the value of the parameter with environmental temperature and pressure at the reference point.

4. In the transition from $\mathrm{K}$ to $\mathrm{M}$, there is an influence of temperature and pressure on the parameter.

A relationship that could be applied to correct the variation in $p_{\mathrm{a}}, T_{\mathrm{a}}$ and $\mathrm{SP}$ in the measurements is defined as:

$\left(\rho_{\mathrm{m}}\right)_{\mathrm{SL}}=\frac{\rho_{\mathrm{m}}}{\delta^{a} \theta^{b} \gamma^{c}}$

where $\left(\rho_{\mathrm{m}}\right)_{\mathrm{SL}}$ is the correct parameter at reference point, $\rho_{\mathrm{m}}$ the measured parameter value at environmental pressure, temperature and rated power, $\delta$ pressure ratio at suction side, $\gamma$ power ratio between shaft power and reference/ design power, $\theta$ temperature ratio between the environmental temperature and reference temperature, $a \delta$ correction exponent, $b \theta$ correction exponent, and $c \gamma$ correction exponent.

In our approach,

$\theta=T_{\mathrm{a}} / 288.15$

$\delta=p_{1} / 0.99$
It should be noted that the reference value for the power is equal to $90 \%$ of maximum rated power. To obtain values for the exponent, $a, b$ and $c$, the TSA has been adopted. In fact, first the influence of the environmental pressure drop is evaluated, then the influence of environmental temperature is considered and finally, the influence of shaft power variation. This process was used to determine the values for the exponents $a, b$ and $c$.

Phase 1: pressure correction exponent $a$

From equation:

$\left(\rho_{\mathrm{b}}\right)_{P}=\frac{\rho_{c}}{\delta^{a}}$

solving:

$a=\frac{\ln \left[\frac{\rho_{\mathrm{c}}}{\left(\rho_{\mathrm{b}}\right)_{P}}\right]}{\ln (\delta)}$

Phase 2: temperature correction exponent $b$

From equation:

$\left(\rho_{\mathrm{b}}\right)_{\mathrm{PT}}=\frac{\left(\rho_{\mathrm{b}}\right)_{P}}{\theta^{b}}$

Analogously:

$b=\frac{\ln \left[\frac{\left(\rho_{\mathrm{b}}\right)_{P}}{\left(\rho_{\mathrm{b}}\right)_{\mathrm{PT}}}\right]}{\ln (\theta)}$

Phase 3: power correction exponent $c$

From equation:

$\left(\rho_{\mathrm{b}}\right)_{\mathrm{SL}}=\frac{\left(\rho_{\mathrm{b}}\right)_{\mathrm{PT}}}{\gamma^{c}}$

Solving by $c$ :

$c=\frac{\ln \left[\frac{\left(\rho_{\mathrm{b}}\right)_{\mathrm{PT}}}{\left(\rho_{\mathrm{b}}\right)_{\mathrm{SL}}}\right]}{\ln (\gamma)}$

\subsection{Level 2}

Once obtained the exponents, the parameter measured, $\rho_{\mathrm{m}}$, has been corrected, by the equation,

$\Delta \rho=\left[\left(\rho_{\mathrm{m}}\right)_{\mathrm{SL}}-\left(\rho_{\mathrm{b}}\right)_{\mathrm{SL}}\right] /\left(\rho_{\mathrm{b}}\right)_{\mathrm{SL}} 100$

The levels 1 and 2 are performed for all measured parameters. The correct parameters are then normalized, as shown in Eq. (12). 


\section{Conclusions}

An ANN model has been exposed. It can be used to perform four basic functions in a gas turbine plant: to generate performance parameters, estimate the plant/process operating conditions, estimate the sensor data and, finally, validate sensors data using the separation of incorrect/noise measurements from good ones.

The various types of ANN currently available are various types of RNA, and some of the most popular include multilayer perceptions, perceptions, radial basis functions, learning vector quantization. Networks can be classified into controlled and uncontrolled or feed forward or feedback based on how the data are processed through the network.

At the end of this first phase, the "correction factors" for the proposal ANN method have been calculated. The next step will be to apply this methodology and calculated correction factors in gas turbine plant, to evaluate the choices and verify the correction introduced, with the aim to separate the noise/incorrect measures from the correct ones.

\section{References}

Agrawal RK, MacIsaac BD, Saravanamuttoo HIH (1978) An analysis procedure for validation of on-site performance measurements of gas turbines. ASME J Eng Power (78-GT-152)

Aker GF, Saravanamuttoo HIH (1989) Predicting gas turbine performance behavior due to compressor fouling using computer simulation techniques. ASME J Eng Gas Turbines Power 111:343-350

Ali M, Gupta U (1990) An expert system for fault diagnosis in a space shuttle main engine. In: 26th AIAA/SAE/ASME/ASEE joint propulsion conference, Orlando, FL, USA, July 16-18, AIAA 90-1890. doi:10.2514/6.1990-1890

Allwood RJ, Kings SP, Pitts NJ (1996) The automatic interpretation of vibration data. Aeronaut J 100(993):99-107

Anderson D, McNeill G (1992) Artificial neural networks technology. Data and analysis center for software (DACS), technical reports. Contract no. F30602-89-C-0082

Angelakis C, Loukis EN, Pouliezos AD, Stavrakakis GS (2001) A neural network based method for gas turbine blading fault diagnosis. Int J Model Simul 21(1):51-60

Bakal B, Adali T, Fakory R, Sonmez MK, Tsaoi O (1995) Neural network simulation of real time core neutronic model. In: Proceedings of the SCS simulation multiconference, Phoenix, AZ, USA

Barlett EB, Uhrig RE (1992) Power plant status diagnostics using artificial neural network. J Nucl Technol 97:272-281

Barschdorff D (1991) Comparison of neural and classical decision algorithms. In: IFAC/IMACS symposium on fault detection, supervision and safety for technical processes. September 10-13. Baden-Baden, Germany

Capata R (2015) Experimental tests of the operating conditions of a micro gas turbine device. Energy Power Eng 9(2015):326-335. doi:10.17265/1934-8975/2015.04.002

Centeno P, Egido I, Domingo C, Fernández F, Rouco L, González M (2005) Review of gas turbine models for power system stability studies. http://aedie.org/9CHLIE-paper-send/368-Centeno.pdf

Colombo P, Pretolani F, Aurora C (2003) Development of a MATLAB model of combined cycle plant to be used in an environment of multivariable predictive control. Technical report (In Italian) within the CESI Contract \# 71/00298EVINGEN/PRESTGEN/2003/01. Pavia (IT)

Hajagos LM, Bérubé GR (2001) Utility experience with gas turbine testing and modeling. In: IEEE power engineering society winter meeting, 2001, Jan 28-Feb 1. doi: 10.1109/PESW.2001.916934

Ogaji SOT (2003) Advanced gas-path fault diagnostics for stationary gas turbines. Ph.D. Thesis, Cranfield University, School of Engineering

Shalan HEMA, Moustafa Hassan MA, Bahgat ABG (2010) Comparative study on modelling of gas turbines in combined cycle power plants. In: Proceedings of the 14th international middle east power systems conference (MEPCON'10), Cairo University, Egypt, December 19-21, 2010, paper id 317 